\title{
Transcatheter Closure of a Ruptured Sinus of Valsalva Aneurysm
}

\author{
Chi-Wei Chang, MD; Shuenn-Nan Chiu, MD; \\ En-Ting Wu, MD; Shen-Kou Tsai, MD, PhD*; \\ Mei-Hwan $\mathrm{Wu}, \mathrm{MD}, \mathrm{PhD}$; Jou-Kou Wang, $\mathrm{MD}, \mathrm{PhD}$
}

\begin{abstract}
Background Ruptured sinus of Valsalva aneurysms (RSVA) can be associated with ventricular septal defects or isolated lesions. Surgical repair has been the traditional treatment of for the RSVA. The results of transcatheter closure of the RSVA in 4 patients are reported.

Methods and Results From 2003 to 2004, 4 patients (2 males and 2 females) aged from 18 years to 47 years with RSVA were identified. The diagnosis of RSVA was made based on a combination of several imaging modalities. The drainage site of the RSVA was right ventricle in 2, and right atrium in remaining 2. All patients underwent general anesthesia and transesophageal echocardiographic (TEE) monitoring during the procedure. Transcatheter closure with an Amplatzer duct occluder was attempted in all 4 patients. The size of the Amplatzer duct occluder selected was up to $2 \mathrm{~mm}$ larger than the maximal diameter of the aortic opening site of the RSVA as measured on TEE images. The attempt to deploy an Amplatzer duct occluder was successful in 3 and a Gianturco coil was deployed in 1. Follow-up (3-18 months) echocardiography showed neither residual shunt nor aortic regurgitation in any of the patients.
\end{abstract}

Conclusion The transcatheter technique is a safe alternative in the treatment of RSVA; however, a longer follow-up is mandatory. (Circ J 2006; 70: 1043-1047)

Key Words: Amplatzer duct occluder; Sinus of Valsalva aneurysm; Transcathter

$\mathbf{R}$ uptured sinus of Valsalva aneurysms (RSVA) are rare and usually occurs in adolescence to early adulthood! They are most commonly congenital in origin and caused by a weakness of the wall of the sinus Valsalva above the aortic valve annulus and leads to progressive dilatation, eventually rupturing into one of the 4 chambers of the heart or the mediastinum? The aneurysm most commonly arises from the right coronary sinus (RCS) of Valsalva and most frequently ruptures into the right ventricle with a wind-sock like structure projecting from the sinus into which it ruptures. Surgical repair is the traditional treatment for RSVA? With the advent of the transcatheter technique, transcatheter closure of the RSVA could be an alternative treatment. We report the results of transcatheter treatment of RSVA in 4 patients.

\section{Methods}

From 2003 to 2004, 4 patients (2 males and 2 females) aged from 18 to 47 years with RSVA were identified. One patient with a ventricular septal defect (VSD) and severe aortic regurgitation due to infective endocarditis underwent VSD repair and aortic valve replacement with mechanical valve (St Jude no. 23). The rest of the patients did not exhibit any intracardiac anomalies. Three patients presented

(Received December 16, 2005; revised manuscript received May 1, 2006; accepted June 1, 2006)

Department of Pediatrics, *Anesthesiology, National Taiwan University Hospital, School of Medicine, National Taiwan University, Taipei, Taiwan

Mailing address: Jou-Kou Wang, MD, PhD, Department of Pediatrics, National Taiwan University Hospital, 7 Chung-Shan S. Rd, Taipei, 100, Taiwan. E-mail: jkw@ha.mc.ntu.edu.tw with sudden-onset exertional dyspnea and palpitation. Bounding pulses and a continuous murmur could be detected in all patients. The diagnosis of RSVA is based on transthoracic 2-dimensional (D) echocardiography with color Doppler and angiography. Two patients had a cardiac chamber dilatation. All 4 patients underwent attempted transcatheter closure of the RSVA after obtaining consent from the patient or parents.

\section{Procedures}

Under local anesthesia, the femoral vein and artery were cannulated with a sheath. Heparin $(50$ units $/ \mathrm{kg}$ ) was administered before the procedure and one dose of cephradine arginine was given. Hemodynamic studies and angiography were performed in all patients to calculate the pulmonaryto-systemic ratio (Qp:Qs) and to delineate the morphology of RSVA and the opening site (Fig 1). After general anesthesia, transesophageal echocardiography (TEE) was performed to monitor the procedure. The maximal diameter of the aortic opening of the RSVA was measured on multiplane TEE images. The distance between the aortic opening site of the RSVA and the right or left coronary ostium was also measured on TEE images. A 5 or $6 \mathrm{Fr}$ right Judkin catheter was advanced from the right femoral artery to the ostium of the RSVA. A 0.014 coronary extra-support guidewire (Boston Scientific, SCIMED, Natick, MA, USA) with a soft and floppy end was advanced through the catheter into the right atrium or the main pulmonary artery. A 10-mm Goose Neck Snare (Microvena, White Bear Lake, MN, USA) was advanced through the right femoral venous sheath, snared the coronary guidewire in the right atrium or the main pulmonary artery, and exteriorized the coronary wire through the right femoral vein. An arteriovenous rail 

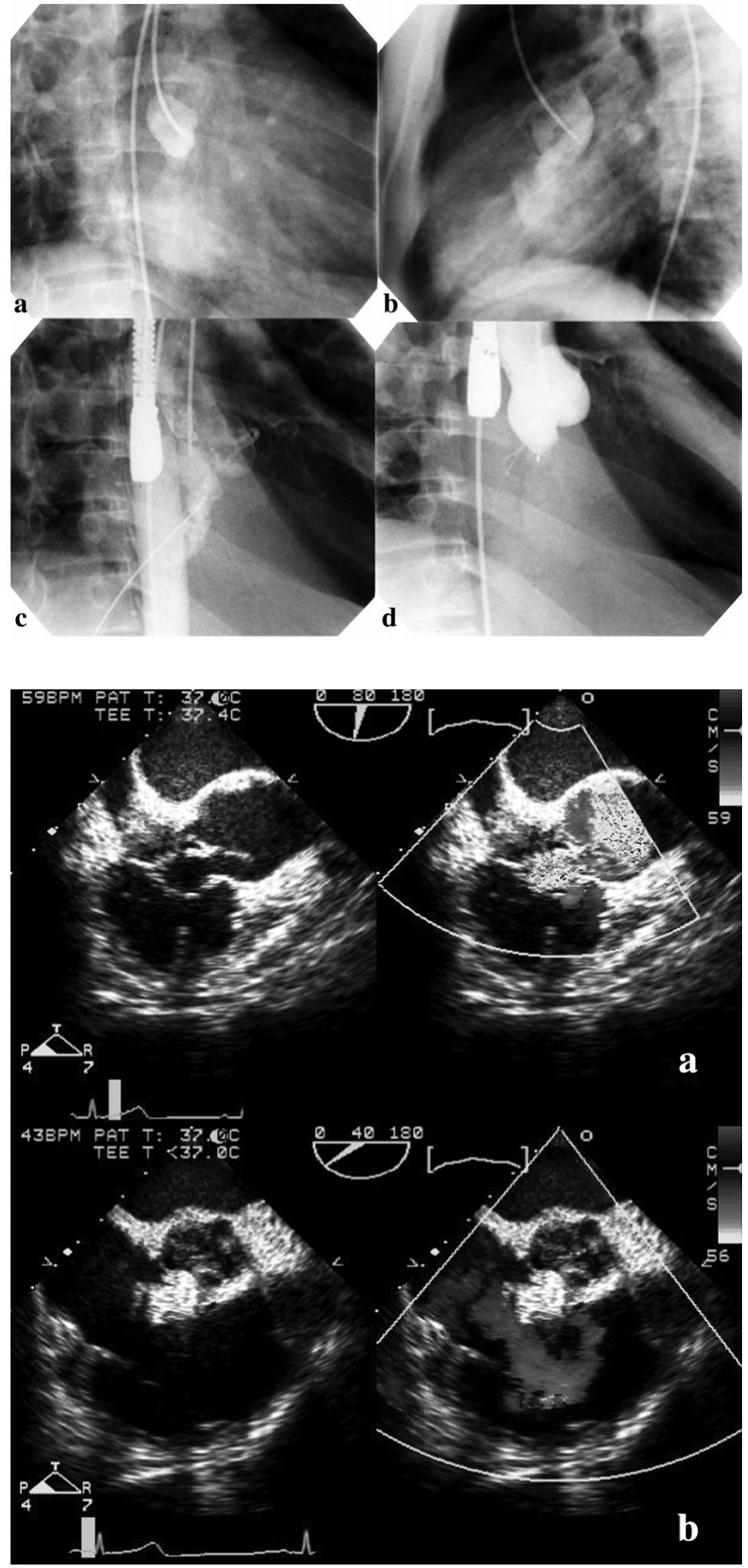

Fig 1. Aortogram showing an aneurysm of the right coronary sinus rupturing into the right atrium: (a) posteroanterior projection; (b) lateral projection. (c) Successful deployment of the Amplatzer duct occluder at the aortic opening of the ruptured sinus of Valsalva aneurysm. (d) The follow-up angiography revealed no residual flow.
Fig 2. Transesophageal echocardiography of the ruptured sinus of Valsalva aneurysm originating from the right coronary sinus. (a) Before Amplatzer duct occluder deployment, a continuous flow from aorta to right atrium. (b) After deployment of an Amplatzer duct occluder, no flow passes through the aneurysm. 


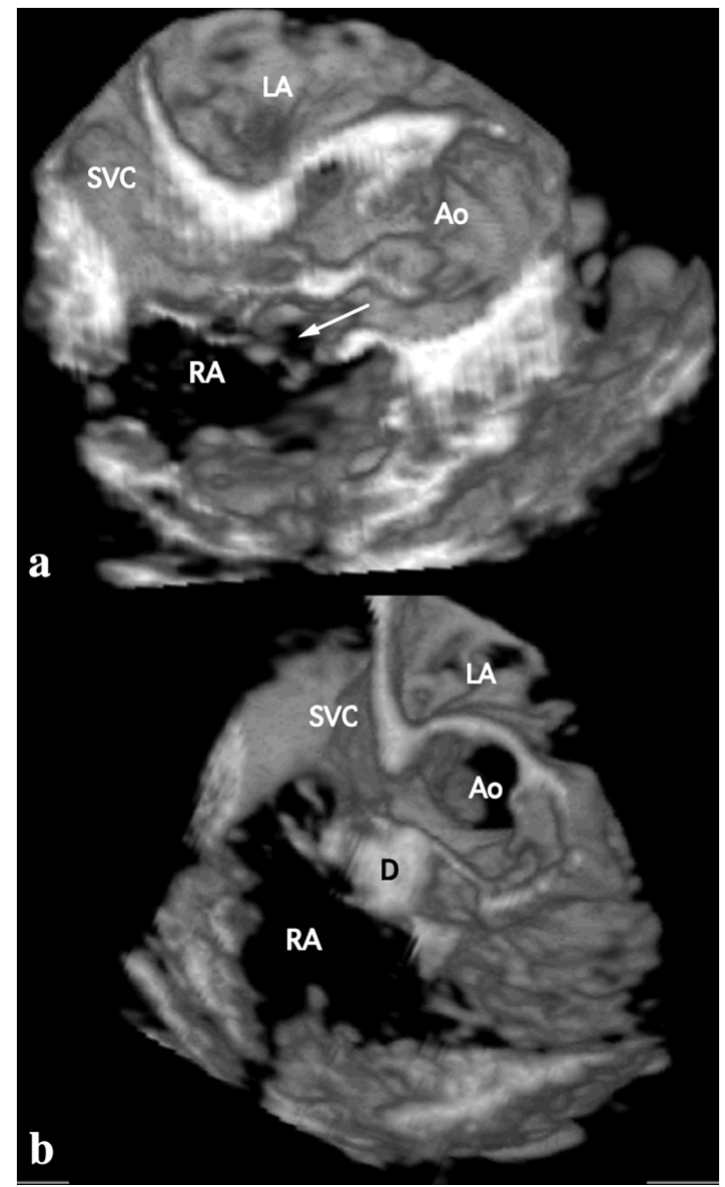

Fig 3. Real-time 3-dimensional transesophageal echocardiography for the ruptured sinus of Valsalva aneurysm from the right coronary sinus to right atrium. (a) Before Amplatzer duct occluder deployment. (b) After device deployment. LA, left atrium; Ao, ascending aorta; RA, right atrium; SVC, superior vena cava; D, Amplatzer duct occluder.

was then established. A 6 or 7Fr delivery sheath (AGA Medical, Golden Valley, MN, USA) was then advanced from the femoral vein to the ascending aorta along the guide wire. An Amplatzer Duct Occluder (AGA Medical) 1$2 \mathrm{~mm}$ larger than the maximal diameter of the aortic opening of the RSVA measured on TEE images was advanced along the AGA sheath. The retention disc was opened in the ascending aorta and then was pulled back to anchor at the RSVA aortic opening site using the TEE for guidance. Then, the rest of the device was deployed in the tunnel. The position of the device was confirmed with both angiography
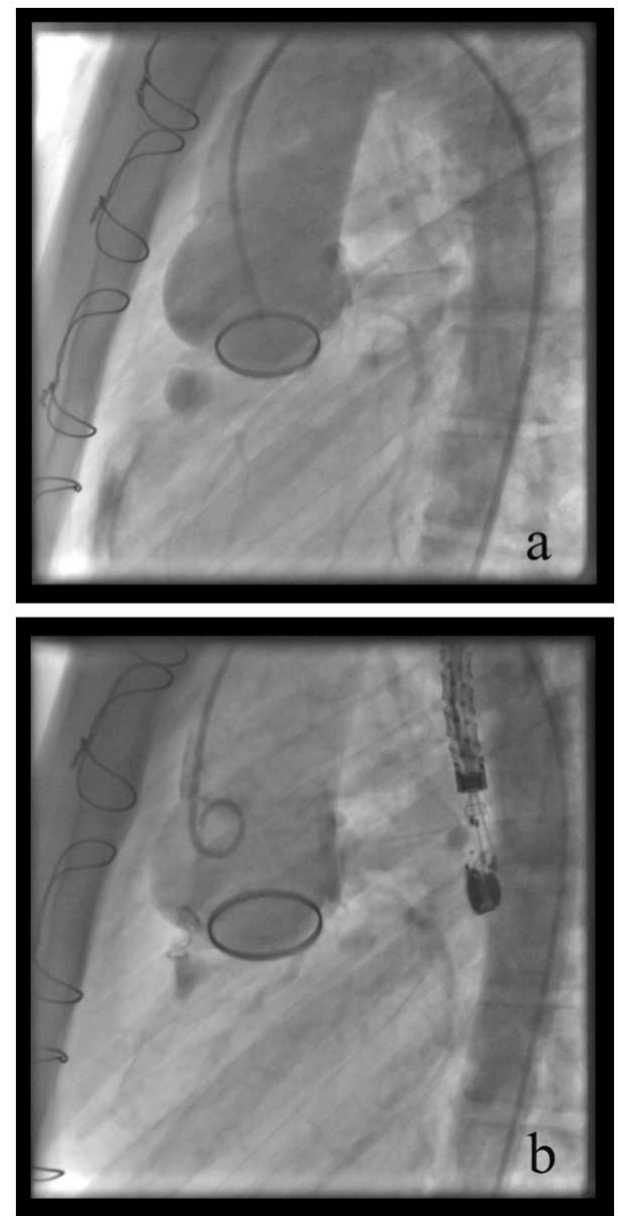

Fig 4. (a) Aortogram showing an aneurysm of the right coronary sinus rupturing into the right ventricle. (b) Successful deployment of a Gianturco coil at the aortic opening of the ruptured sinus of Valsalva aneurysm and follow-up angiography showing slight residual flow from the aorta to right ventricle.

and TEE (2-D and real-time 3-D) to ensure that the aortic valve and coronary ostium were not compromised before detaching the device from the delivery cable (Figs 2,3). A repeated aortogram was performed after the detachment to evaluate the position of the device, residual shunt and aortic valve function (Fig 1). All patients received another 3 doses of antibiotics thereafter. All patients received aspirin $(3-5 \mathrm{mg} / \mathrm{kg}$ per day) or platelet-inhibiting agent (Plavix) for a 6-month period and follow-up transthoracic echocardiography was undertaken at intervals of 1,3 and 6 months after the procedure.

Table 1 Characteristics of the 4 Patients Undergoing Transcatheter Closure of RSVA

\begin{tabular}{cccccccccc}
\hline \hline $\begin{array}{c}\text { Case } \\
\text { no. }\end{array}$ & $\begin{array}{c}\text { Age } \\
\text { (years) }\end{array}$ & Sex & $\begin{array}{c}\text { Change in pulse } \\
\text { pressure }(\mathrm{mmHg}) \\
\text { Pre } \rightarrow \text { Post }\end{array}$ & $\begin{array}{c}\text { Qp:Qs } \\
\text { ratio }\end{array}$ & Site & $\begin{array}{c}\text { Diameter of } \\
\text { aortic opening } \\
\text { of RSVA (mm) }\end{array}$ & Device & $\begin{array}{c}\text { Residual } \\
\text { shunt }\end{array}$ & $\begin{array}{c}\text { PT/FT } \\
\text { (min) }\end{array}$ \\
\hline 1 & 22 & $M$ & $56 \rightarrow 39$ & 2.2 & NCSV to RA & 8 & ADO (12/10) & Nil & $65 / 23$ \\
2 & 47 & $F$ & $65 \rightarrow 58$ & 2 & RCSV to RA & 7 & ADO (10/8) & Nil & $60 / 7.5$ \\
$3 *$ & 18 & $M$ & $41 \rightarrow 30$ & 1.4 & RCSV to $R V$ & 4 & Coil $38-8-5$ & Small & $200 / 52$ \\
4 & 22 & $F$ & $53 \rightarrow 31$ & 2.6 & RCSV to RV & 8 & ADO (10/8) & Nil & $82 / 19$ \\
\hline
\end{tabular}

*After VSD repair and aortic valve replacement.

Qp:Qs ratio, calculate the pulmonary-to-systemic ratio; RSVA, ruptured sinus of Valsalva aneurysms; PT, procedure time; FT, fluoroscopic time (min); NCSV, noncoronary sinus Valsalva; RA, right atrium; ADO, amplatzer duct occluder; RCSV, right coronary sinus Valsalva; $R V$, right ventricle; VSD, ventricular septal defect. 


\section{Results}

The clinical information of all 4 patients is summarized in Table 1. Two patients had RSVA from the RCS to the right ventricle, one had ruptured the non-coronary sinus to the right atrium and another had ruptured the RCS to the

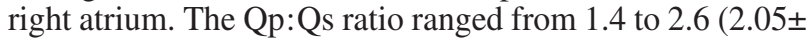
$0.5)$. The diameter of the RSVA aortic opening measured on the TEE images ranged from 4 to $8 \mathrm{~mm}(6.75 \pm 1.9 \mathrm{~mm})$. The deployment of the Amplatzer duct occluder was successful in 3 patients but failed in 1 . In the latter patient, the AGA delivery sheath could not negotiate the curvature between the right ventricle and the RSVA drainage site. Therefore, a right Judkin catheter was advanced retrogradely from the aorta to the RSVA. To prevent coil migration and distal embolization, a Gianturco coil (MWCE 38-8-5) (Cook, Bloominton, IN, USA) with a diameter twice the maximal diameter of the aortic opening of the RSVA, as measured using TEE images, was selected. One loop of the coil was delivered into the RSVA, and the remaining loops were delivered in the RCS (Fig 4). Although a small residual shunt was detected on the follow-up angiography, the procedure was terminated because the 0.035 floppy guidewire could not pass through the aortic opening to the RSVA. Following the procedure, the mean aortic pulse pressure decreased by $14.3 \pm 6.6 \mathrm{mmHg}$ (ranging from 7 to $22 \mathrm{mmHg}$ ). The procedure time ranged from 60 to $200 \mathrm{~min}$ $(101.25 \pm 66.3 \mathrm{~min})$ and the fluoroscopic time ranged from 7.5 to 52 mins $(25.4 \pm 18.9$ mins $)$. The 1 -day follow-up echocardiography showed the device was sitting well in the aneurysm and there was no leakage. None of the patients developed hemolysis, but one patient complained of palpitation on the following day. An electrocardiogram showed sinus arrhythmia with occasional bradycardia of about 49 to 58 beats/min and some ventricular premature complex. A sinus node function test showed normal sinus function. Holter monitoring revealed normal sinus rhythm and frequent ventricular premature complex. During the follow-up period (3-18 months), all 4 patients experienced an improvement in their symptoms. Echocardiography in the latest follow up showed neither residual shunts nor aortic insufficiencies.

\section{Discussion}

The congenital aneurysm of the sinus of Valsalva accounts for 0.1 to $3.5 \%$ of congenital heart defects.,5 A racial predominance is suggested by the finding that the incidence of sinus of Valsalva aneurysms is higher in Asians than in Caucasians, 4 RSVA is frequently associated with other congenital defects such as VSD, aortic insufficiency or bicuspid aortic valve 7,8 Sinus of Valsalva aneurysms may occur in the right, left or non-coronary sinus, where the RCS is the most common (75-90\%), followed by the noncoronary sinus (10-25\%), with the reminder occurring in the left coronary sinus? The RSVA drainage sites can be single or multiple, and the right ventricle was most common drainage site!

It has been postulated that the defect results from the incomplete fusion of the distal bulbar septum (primitive bulbis cordis) and truncal ridges, which leads to a weakness between the aortic annulus and aortic media corresponding to the right aortic sinus and right half of the non-coronary sinus. This may result in the formation of the aneurysmal sac. The walls of sinus of Valsalva aneurysm are composed of fibrous tissue!,2,4 The sac appears as a windsock-like projection and is the result of continuing high aortic pressures on the weakened tissues!

Acquired sinus of Valsalva aneurysms are less commonly reported than congenital ones and are usually associated with acquired heart diseases such as syphilis or bacterial endocarditis, direct trauma and surgical complications during surgical repair of a VSD, or removal of a calcified aortic valve and mitral valve! ${ }^{10}$

The sinus of Valsalva aneurysm is usually clinically silent until it ruptures. The most common age of rupture of sinus Valsalva aneurysm is from adolescence to early adulthood! The common symptoms include sudden onset of dyspnea, fatigue and paroxysmal nocturnal dyspnea or orthopnea due to abrupt volume overload caused by a significant left-to-right shunt. The symptoms are generally precipitated by physical stress. Chest pain is reported in few cases, ${ }^{4}, 11$

Surgical repair is the conventional treatment for the RSVA with an operative mortality rate less than $2 \%$ ! The long-term surgical outcomes for RSVA are generally good with $85 \%$ patients in New York Heart Association Functional Class I or II?,6,12 Vural et al proposed an algorithmdependent classification for the timing for surgical repair!3

Recently the transcatheter technique has been used to close RSVA. Cullen et al reported the first case of RSVA closure with a Rashkind umbrella in $1994 !^{14}$ Rao et al reported the use of a Gianturco coil 0.052" with a bioptome assisted technique to close RSVA in one ${ }^{15}$ Fedson et al reported use of the Amplatzer duct occluder in closing the RSVA in a patient in whom the retention disc was deployed in the aneurysm and the rest of the device to the drainage site ${ }^{16}$ Arora et al reported a series of 8 patients with RSVA, who underwent transcatheter closure with Amplatzer duct occluder in 5, an Amplatzer septal occluder in 1 and a Rashkind umbrella device in $2 !^{17}$

In our patients, the retention disc of the Amplatzer duct occluder was deployed in the sinus of Valsalva instead of in the aneurysm because a progressive dilatation of the aneurysm may develop if the retention disc is deployed within the aneurysm. Multiple drainage sites could be present in RSVA. The retention disc should be deployed in the aortic opening site of the sinus of Valsalva aneurysm to avoid the use of multiple devices. The maximal diameter of the aortic opening of the aneurysm can be measured on both TEE images and angiograms. The balloon sizing of the diameter of the aortic opening site was performed in Arora et al series ${ }^{17}$ In our series, the size of Amplatzer duct occluder selected was up to $2 \mathrm{~mm}$ larger than the maximal diameter of the aortic opening of RSVA as measured on the TEE images. The procedure should be monitored using TEE images to ensure the position of the device and to minimize the risk of impingement on the aortic valve or coronary ostium. Before the detachment of the device, the position of the Amplatzer duct occluder should be checked on both the TEE images and an angiogram for the possibility of compromising the aortic valve opening and obstructing of the coronary ostium. Real-time 3-D echocardiography may be a useful tool in this regard. ${ }^{18}$

In patient 3 , the drainage site of the RSVA was the right ventricle. There was an acute curve between the right ventricle and drainage site of RSVA, which prevented the advancement of the AGA delivery sheath. Therefore, a Gianturco coil was deployed via the aortic route. Although, the Amplatzer duct occluder device had advantages in 
terms of repositionability, stability and a higher complete occlusion rate, coil closure is an alternative when the RSVA can not be approached using the AGA Medical delivery system via a venous route. However, most RSVA are so large that coil closure carries a high risk of distal embolization. The drainage course of the RSVA in patient No. 3 was so tortuous that coil closure was considered safer. Similar to the closure technique for patent ductus arteriosus, the coil diameter selected should be at least twice the diameter of the RSVA aortic opening and one loop of the coil should be delivered in the aneurysm and the rest of the loops in the sinus of Valsalva.

Because RSVA may have multiple drainage sites, the occurrence of new rupture sites in the same sinus of Valvalva after transcatheter closure is possible! The aortic valve function, coronary blood flow and development of thromboembolism should be carefully evaluated during follow-up.

\section{Conclusion}

The transcatheter technique provides good efficacy in the treatment of RSVA and avoids the risks of open heart surgery and cardiopulmonary bypass. Therefore, the transcatheter technique could be an alternative to surgery in the treatment of RSVA; however, a longer follow-up evaluation is mandatory.

\section{References}

1. Goldberg N, Krasnow N. Sinus of Valsalva aneurysm. Clin Cardiol 1990; 13: 831-836.

2. Edwards E, Burchell HB. The pathological anatomy of deficiencies between the aortic root and the heart, including aortic sinus aneurysms. Thorax 1957; 12: 125-139.

3. Barragry TP, Ring WS, Moller JH, Lillehei CW. Fifteen-to-thirtyyear follow-up of patients undergoing repair of ruptured congenital aneurysms of the sinus of Valsalva. Ann Thorac Surg 1988; 46: $515-$ 519.
4. Taguchi K, Sasaki N, Matsura Y, Uemura R. Surgical correction of aneurysms of the sinus Valsalva: A report of forty-five consecutive patients including eight with total replacement of the aortic valve. Am J Cardiol 1969; 23: 180-191.

5. McGoon DC, Edwards JE, Kirklin JW. Surgical treatment of ruptured aneurysm of aortic sinus. Ann Thorac Surg 1958; 147: 387-392.

6. Abe T, Komatsu S. Surgical repair and long term results of ruptured sinus of Valsalva aneurysm. Ann Thorac Surg 1988; 46: 520-535.

7. van Son JA, Danielson GK, Schaff HV, Orszulak TA, Edwards WD, Seward JB. Long-term outcome of surgical repair of ruptured sinus of Valsalva aneurysm. Circulation 1994; 90: II-20-II-29.

8. Davis JA, Cecchin F, Jones TK, Portman MA. Major coronary artery anomalies in a pediatric population: Incidence and clinical importance. J Am Coll Cardiol 2001; 37: 593-597.

9. Fishbein MC, Obma R, Roberts WC. Unruptured sinus of Valsalva aneurysm. Am J Cardiol 1975; 35: 918-922.

10. Sokol DM, Trachtenberg J, Goldman BS, Mustard WT. Surgical experience with ruptured aneurysms of the sinuses of Valsalva. Chest 1973; 64: 615-618.

11. Buckley B, Hutchins GM, Ross RS. Aortic sinus of Valsalva aneurysms stimulating primary right-sided heart disease. Circulation 1975; 52: 696-699.

12. Naka Y, Kadoba K, Ohtake S, Sawa Y, Hirata N, Nishimura M, et al. The long-term outcome of a surgical repair of sinus of Valsalva aneurysm. Ann Thorac Surg 2000; 70: 727-729.

13. Vural KM, Sener E, Tasdemir O, Bayazit K. Approach to sinus of Valsalva aneurysm: A review of 53 cases. Eur J Cardiothorac Surg 2001; 20: 71-76.

14. Cullen S, Somerville J, Redington A. Transcatheter closure of a ruptured aneurysm of the sinus Valsalva. Br Heart J 1994; 71: 479480.

15. Rao PS, Bromberg BI, Jureidini SB, Fiore AC. Transcatheter occlusion of ruptured sinus of valsalva aneurysm: Innovative use of available technology. Catheter Cardiovasc Interv 2003; 58: 130-134.

16. Fedson S, Jolly N, Lang RM, Hijazi ZM. Percutaneous closure of a ruptured sinus of Valsalva aneurysm using the Amplatzer Duct Occluder. Catheter Cardiovasc Interv 2003; 58: 406-411.

17. Arora R, Trehan V, Rangasetty UM, Mukhopadhyay S, Thakur AK, Kalra GS. Transcatheter closure of ruptured sinus of valsalva aneurysm. J Interv Cardiol 2004; 17: 53-58.

18. Jean WH, Kang TJ, Liu CM, Chang CW, Tsai SK, Wang JK. Transcatheter occlusion of ruptured sinus of Valsalva aneurysm guided by three-dimensional transesophageal echocardiography. J Formos Med Assoc 2004; 103: 948-951. 